\title{
Urinary kallidinogenase for the treatment of cerebral arterial stenosis
}

\author{
This article was published in the following Dove Press journal: \\ Drug Design, Development and Therapy \\ 13 October 2015 \\ Number of times this article has been viewed
}

\author{
Liandong Zhaol,2 \\ Ying Zhao ${ }^{2}$ \\ Qi Wan' \\ Haijun Zhang ${ }^{3}$
}

'Department of Neurology, The First Affiliated Hospital of Nanjing Medical University, Nanjing, ${ }^{2}$ Department of Neurology, The Second People's Hospital of Huai'an and The Affiliated Huai'an Hospital of Xuzhou Medical College, Huai'an, Jiangsu, ${ }^{3}$ Department of Oncology, Zhongda Hospital,

Medical School, Southeast University, Nanjing, People's Republic of China

Correspondence: Qi Wan

Department of Neurology, The First Affiliated Hospital of Nanjing Medical University, Guangzhou Road 300, Nanjing 210029, People's Republic of China

Tel +86 25 837I 45 I I

Fax +86 2586260332

Email wanqisry@sina.com

Haijun Zhang

Department of Oncology, Zhongda Hospital, Medical School, Southeast University, 87 Dingjiaqiao, Nanjing 210009 , People's Republic of China Tel/fax +8625832754I8

Email zhanghaijunseu@।63.com
Aim: Urinary kallidinogenase (UK) has shown promise in improving cerebral perfusion. This study aimed to examine how UK affects cognitive status and serum levels of amyloid betas (A $\beta$ s) 1-40 and 1-42 in patients with cerebral arterial stenosis.

Methods: Ninety patients with cerebral arterial stenosis were enrolled, of whom 45 patients received UK + conventional treatment (UK group), and 45 patients received conventional treatment alone as control group. Cognitive status and $A \beta 1-40$ and $A \beta 1-42$ serum levels were determined before treatment and at 4 weeks and 8 weeks after treatment.

Results: At 4 weeks after treatment, cognitive status in patients treated with UK clearly improved accompanied by $A \beta 1-40$ serum levels decreasing while there was no change of $A \beta 1-42$. Cognitive status in patients receiving UK continued to improve, $A \beta 1-40$ serum levels declined further as well as $A \beta 1-42$ serum levels began to decrease dramatically at 8 weeks after treatment.

Conclusion: UK could improve cognitive status and decrease both $A \beta 1-40$ and $A \beta 1-42$ serum levels to prevent ischemic cerebral injury, which represents a good option for patients with cerebral arterial stenosis.

Keywords: urinary kallidinogenase, arterial stenosis, Alzheimer's disease, A $\beta 1-40, A \beta 1-42$

\section{Introduction}

Urinary kallikrein, a glycoprotein extracted from urine, can activate kallikrein-kinin system (KKS) and thus transfer kininogen hydrolysis into kallidin and kinin. ${ }^{1}$ Kinin in turn could activate $\mathrm{B} 1$ and $\mathrm{B} 2$ receptors induced by ischemic brain tissue and trigger a series of biological effects, releasing nitric oxide and relaxing vascular smooth muscle. ${ }^{1}$ Thus, it can expand blood vessels in the ischemic area, improve cerebral blood supply of penumbra, and restore the neurological deficit. ${ }^{1}$ Urinary kallidinogenase (UK) for injection (Kailikang ${ }^{\circledR}$; Techpool Bio-Pharma Co, Ltd, Guangdong, People’s Republic of China) has been approved by Chinese State Food and Drug Administration and used clinically in People's Republic of China to treat patients with stroke ${ }^{2}$ (molecular formula and structure are shown in Figure 1). In addition, studies on hind limb ischemia, cardiac infarction, and renal ischemia confirmed that kallikrein attempts to maintain an adequate tissue perfusion. ${ }^{3}$ However, whether the commercially available KKS-regulating medicine, UK for injection (Kailikang ${ }^{\circledR}$ ), is capable to improve the outcome of the cerebral arterial stenosis has not been reported yet.

Cerebral arterial stenosis, including intracranial and/or extracranial artery stenosis, is characterized by narrowed arteries that supply blood to the brain, resulting in cerebral hypoperfusion. ${ }^{4}$ Patients with severe cerebral artery stenosis run a higher risk of distal embolization, hemodynamic changes, and sudden vascular occlusion..$^{5-7}$ Alzheimer's disease (AD) is associated with decreased cerebral blood flow. It was found that AD cases displayed a degree of cerebral artery occlusion and that there was a positive 
A Ile-Val-Gly-Gly-Trp-Glu-Cys-Glu-GIn-His-Ser-Gln-Pro-Trp-Gln-Ala-Ala-Leu-Tyr-HisPhe-Ser-Thr-Phe-GIn-Cys-Gly-Gly-Ile-Leu-Val-His-Arg-GIn-Trp-Val-Leu-Thr-Ala-Ala-

His-Cys-Ile-Ser-Asp-Asn-Tyr-GIn-Leu-Trp-Leu-Gly-Arg-His-Asn-Leu-Phe-Asp-Asp-Glu-

Asn-Thr-Ala-GIn-Phe-Val-His-Val-Ser-Glu-Ser-Phe-Pro-His-Pro-Gly-Phe-Asn-Met-Ser-

Leu-Leu-Glu-Asn-His-Thr-Arg-Gln-Ala-Asp-Glu-Asp-Tyr-Ser-His-Asp-Leu-Met-Leu-Leu-

Arg-Leu-Thr-Glu-Pro-Ala-Asp-Thr-Ile-Thr-Asp-Ala-Val-Lys-Val-Val-Glu-Leu-Pro-Thr-

GIn-Glu-Pro-Glu-Val-Gly-Ser-Thr-Cys-Leu-Ala-Ser-Gly-Trp-Gly-Ser-lle-Glu-Pro-Glu-

Asn-Phe-Ser-Phe-Pro-Asp-Asp-Leu-GIn-Cys-Val-Asp-Leu-Lys-Ile-Leu-Pro-Asn-Asp-Glu-

Cys-Glu-Glu-Ala-His-Val-Gln-Lys-Val-Thy-Asp-Phe-Met-Leu-Cys-Val-Gly-His-Leu-Glu-

Gly-Gly-Lys-Asp-Thr-Cys-Val-Gly-Asp-Ser-Gly-Gly-Pro-Leu-Met-Cys-Asp-Gly-Val-Leu-

GIn-Gly-Val-Thr-Ser-Trp-Gly-Tyr-Val-Pro-Cys-Gly-Thr-Pro-Asn-Lys-Pro-Ser-Val-Ala-

Val-Arg-Val-Leu-Ser-Tyr-Val-Lys-Trp-Ile-Glu-Asp-Thr-Ile-Ala-Glu-Asn-Ser

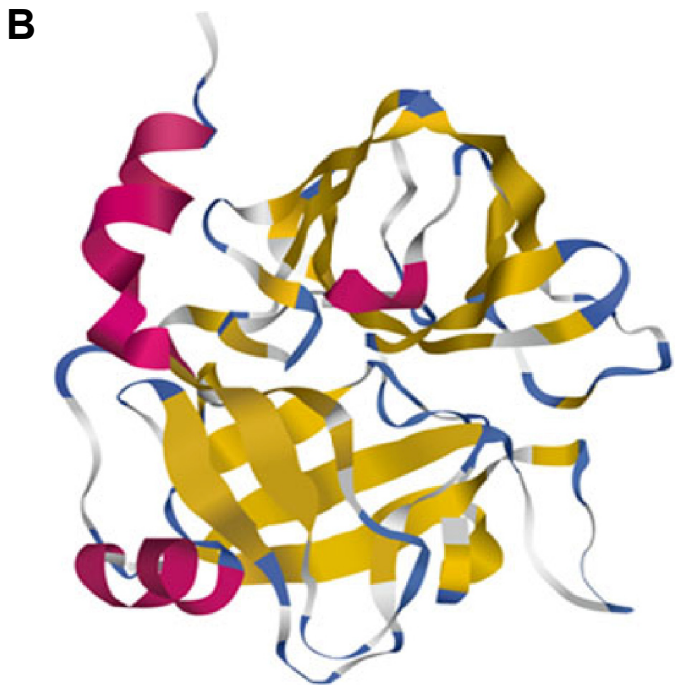

Figure I Molecular formula (A) and structure (B) of urinary kallidinogenase.

correlation between the degree of arterial stenosis and neurofibrillary tangle (NFT) score. ${ }^{8}$ Chronic brain hypoperfusion leading to cerebral hypoxia represents an important aspect of neurodegenerative processes in AD. ${ }^{9}$ The current hypothesis of the pathogenesis of familial AD results mainly from aberrant cleavage of amyloid beta $(\mathrm{A} \beta)$ precursor protein, which produces cytotoxic $A \beta$ fragments, causes progressive degeneration and dysfunction of neurons, and ultimately leads to the development of $\mathrm{AD} .^{4}$ The contribution of $\mathrm{A} \beta$ deposition in the pathogenesis of $\mathrm{AD}$ has been well established, and $\mathrm{A} \beta$ appears to have a role in ischemic cerebral damage. ${ }^{10}$

For patients with cerebral arterial stenosis, improving the cerebrovascular perfusion remains a good option to rescue injured tissue and alleviate neurological deficits. In this study, we evaluated the cognitive status and the serum levels of $A \beta 1-40$ and $A \beta 1-42$ in patients with cerebral arterial stenosis treated with or without UK before treatment and at 4 weeks and 8 weeks after treatment. Our intention was to provide additional evidence on the efficacy of UK in the application of patients with cerebral arterial stenosis.

\section{Materials and methods Patients}

Ninety patients were recruited from the Second People's Hospital of Huai'an from June 1, 2013, to May 31, 2015. The study was approved by the Ethics Committee of the Second People's Hospital of Huai'an, and informed consents were obtained from all the participants. Patients with 
dizziness, transient ischemic attack, and $70 \%$ or more internal carotid artery stenosis on one or both sides as determined by neuroimaging examination such as digital subtraction angiography and magnetic resonance imaging were included in the study. ${ }^{4}$ Patients were excluded if they had a history of major medical illness, such as heart failure, pulmonary insufficiency, renal insufficiency, hepatic dysfunction, or blood diseases unfit for antiplatelet therapy. ${ }^{4}$ Participants were randomly assigned $(1: 1)$ to receive either a UK treatment (UK group) or a conventional treatment (control group) using a computer-generated random sequence. The UK group consisted of 45 patients ( 24 men and 21 women) aged from 42 years to 84 years, including 21 cases of unilateral internal carotid artery stenosis, twelve cases of bilateral internal carotid artery stenosis, eight cases of unilateral internal carotid artery stenosis associated with vertebral artery stenosis, and four cases of unilateral internal carotid artery stenosis associated with middle cerebral artery stenosis. The control group also consisted of 45 patients (26 men and 19 women) aged from 40 years to 79 years, including 19 cases of unilateral internal carotid artery stenosis, eleven cases of bilateral internal carotid artery stenosis, ten cases of unilateral internal carotid artery stenosis associated with vertebral artery stenosis, and five cases of unilateral internal carotid artery stenosis associated with middle cerebral artery stenosis. All patients had experienced at least 1 month of nervous-system dysfunctions. No severe cerebrovascular problems such as stroke occurred during the study period. Clinical symptoms of cerebral ischemia, such as paralysis of limbs and/or disturbance of consciousness, were observed in most of the cases.

\section{Procedures}

The study procedure is shown in Figure 2. During the study period, all patients were given $75 \mathrm{mg}$ of clopidogrel and $100 \mathrm{mg}$ of enteric-coated aspirin orally once a day in addition to the use of salvia, puerarin (Conba Phamaceuticals, Zhejiang, People's Republic of China), and an anti-freeradical agent, edaravone (Lijun Phamaceuticals, Xian, People's Republic of China), as conventional treatment in the control group. In addition, UK was injected into patients in the UK group at a dose of 0.15 paranitroanilinum (PNA)/d every day for 21 days. There was no daily and total effect of a daily injection of UK on blood pressure (BP), pulse rate, and heart rate. Mini-mental state examination (MMSE) scale was performed to evaluate the efficacy of UK on the cognitive status before treatment and at 4 weeks and 8 weeks after treatment in all patients. MMSE scale was scored by experienced

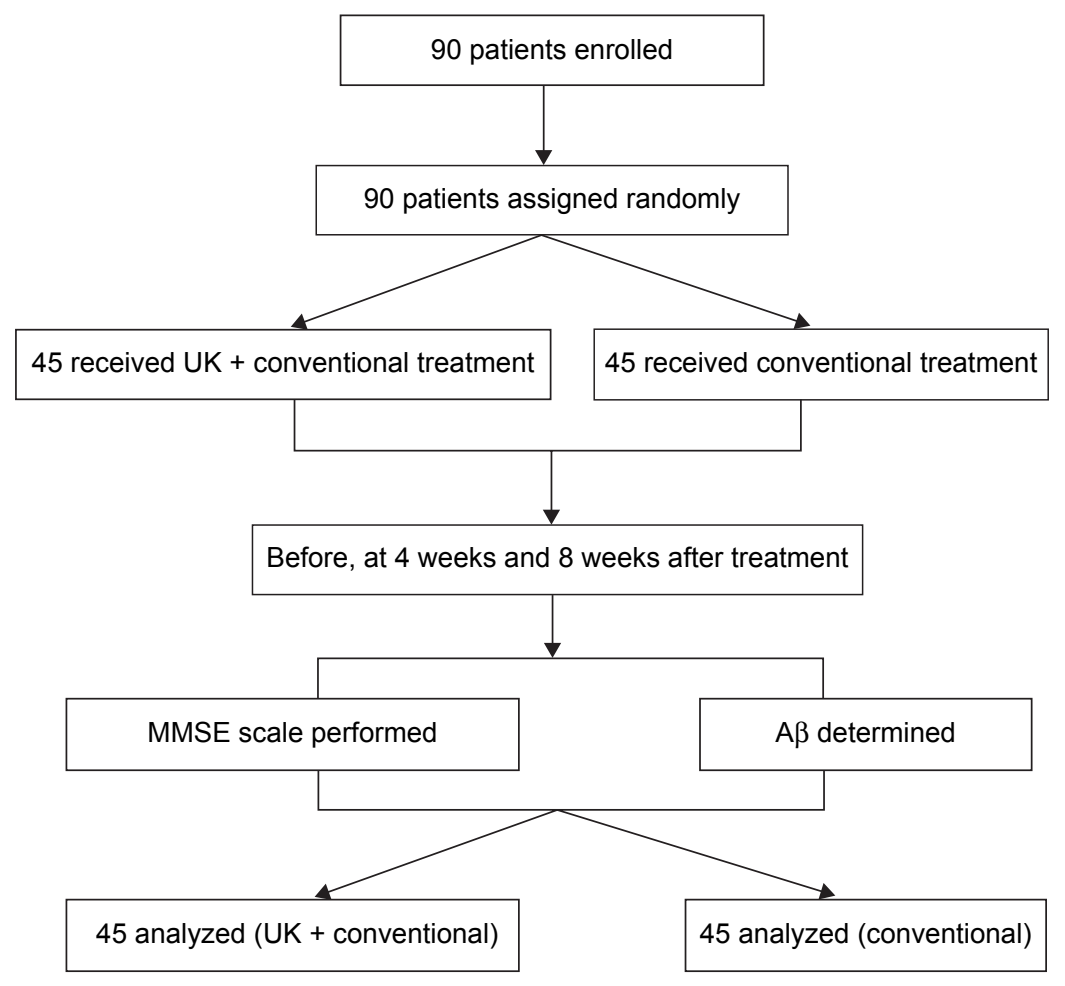

Figure 2 The schedule of the study.

Abbreviations: MMSE, mini-mental state examination; UK, urinary kallidinogenase. 
neurologists, who had over 10 years of experience in professional neuropsychological scale assessment and were blinded to the clinical findings. Moreover, $5 \mathrm{~mL}$ blood samples were collected via cubital vein from patients before treatment and at 4 weeks and 8 weeks after treatment. The blood samples were centrifuged at 1,000 rpm for 10 minutes, and sera were immediately collected and stored at $-70^{\circ} \mathrm{C}$. Then, serum concentrations of $\mathrm{A} \beta$ were determined using solid-phase sandwich enzyme-linked immunosorbent assay kit as per the manufacturer's instructions (Adlitteram Diagnostic Laboratories, San Diego, CA, USA). Furthermore, the evaluator of $\mathrm{A} \beta$ was blind to the results of grouping.

\section{Statistical analysis}

Statistical analyses were performed using SPSS software (Version 10.0; IBM Corporation, Armonk, NY, USA). Continuous variables were expressed as mean \pm standard deviation, whereas categorical variables were expressed as percentage. The $F$-test was used for significance testing of continuous variables, and the $\chi^{2}$ statistic was used for testing of categorical variables. $P<0.05$ was considered to be statistically significant.

\section{Results}

\section{Characteristics of enrolled patients}

As shown in Table 1, 90 patients were enrolled, of whom 45 patients received conventional treatment + UK (UK group) and another 45 patients only received conventional treatment (control group), and the two groups were similar in basic characteristics for analysis.

\section{Comparison of MMSE before and after treatment}

To investigate the effect of UK on the cognitive status in patients with cerebral arterial stenosis, we first evaluated

Table I Characteristics of enrolled patients

\begin{tabular}{llll}
\hline Characteristics & UK group & Control group & $P$-value \\
\hline Age (years), (mean \pm SD) & $57.26 \pm 9.35$ & $56.8 I \pm 9.27$ & 0.82 \\
Sex & & & 0.67 \\
$\quad$ Male & 24 & 26 & \\
$\quad$ Female & 21 & 19 & 0.92 \\
Distribution of arterial & & & \\
stenosis & & & \\
$\quad$ Unilateral ICA & 21 & 19 & \\
$\quad$ Bilateral ICA & 12 & 11 & \\
$\quad$ Unilateral ICA + VA & 8 & 10 & \\
$\quad$ Unilateral ICA + MCA & 4 & 5 & \\
\hline
\end{tabular}

Abbreviations: UK, urinary kallidinogenase; ICA, internal carotid artery; VA, vertebral artery; MCA, middle cerebral artery.

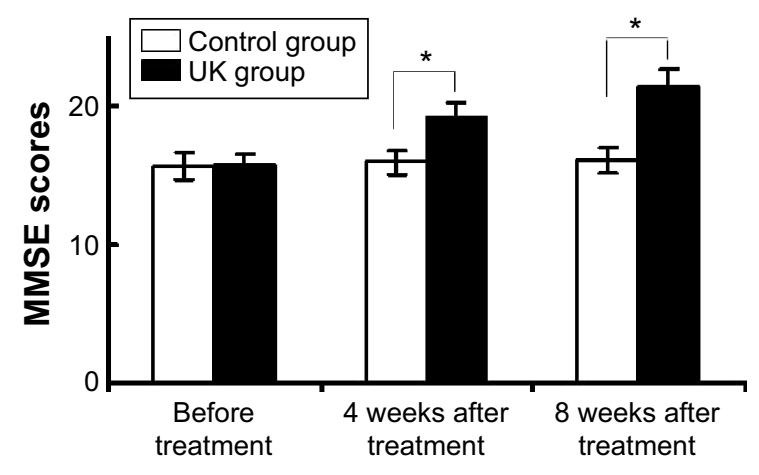

Figure 3 MMSE scores before and after treatment.

Note: $* P<0.05$.

Abbreviations: MMSE, mini-mental state examination; UK, urinary kallidinogenase.

the cognitive status with MMSE scale before any treatment. As shown in Figure 3, there was no obvious difference of the score of MMSE between the UK group and the control group before any treatment. The score of MMSE obviously improved at 4 weeks and 8 weeks after patients treated with UK. However, during the study, the MMSE score of patients with conventional treatment still remained similar to that of patients before treatment.

\section{Effect of UK on serum levels of $A \beta$}

As shown in Table 2, no significant difference was found in the serum levels of $A \beta 1-40$ and $A \beta 1-42$ between the UK group and the control group before treatment. There were no significant changes of both $A \beta 1-40$ and $A \beta 1-42$ in the control group throughout the study. However, the serum levels of A $\beta 1-40$ in the UK group decreased significantly at 4 weeks after treatment and further 8 weeks after treatment. The serum levels of $A \beta 1-42$ in the UK group display different manners than that of $A \beta 1-40$ that began to decrease significantly at 8 weeks after treatment.

\section{Discussion}

Perfusion and hypoxia due to cerebral arterial stenosis may promote $\beta$-site cleavage of $A \beta$ precursor protein and cleavage activity of $\gamma$-secretase, resulting in increased levels of various $A \beta$ fragments such as $A \beta 1-40$ and $A \beta 1-42$ in the brain. ${ }^{4}$ An imbalance between production and clearance of $\mathrm{A} \beta$ fragments may cause abnormal accumulation of these peptides inside the brain and the subsequent development of AD. ${ }^{11-13}$ Various mechanisms are involved in the detrimental effects of $A \beta$. For example, $A \beta$ could activate astrocytes and microglias and cause inflammatory reactions via stimulating expression and release of tumor necrosis factor-alpha, interleukin-1, interleukin-6, and NO. ${ }^{14,15} \mathrm{~A} \beta$ might also induce nerve-cell apoptosis and disrupt the 
Table 2 Effect of UK on serum levels of amyloid beta

\begin{tabular}{|c|c|c|c|c|}
\hline$A \beta$ & Group & $\begin{array}{l}\text { Before } \\
\text { treatment (ng/L) }\end{array}$ & $\begin{array}{l}4 \text { weeks after } \\
\text { treatment }(\mathrm{ng} / \mathrm{L})\end{array}$ & $\begin{array}{l}8 \text { weeks after } \\
\text { treatment (ng/L) }\end{array}$ \\
\hline \multirow[t]{2}{*}{$A \beta I-40$} & UK & $1.25 \pm 0.29$ & $\mathrm{I} .0 \mathrm{I} \pm 0.22^{* * * *}$ & $0.87 \pm 0.18^{* * * *, * * *}$ \\
\hline & Control & $1.24 \pm 0.33$ & I. $14 \pm 0.27$ & $1.16 \pm 0.24$ \\
\hline \multirow[t]{2}{*}{$A \beta 1-42$} & UK & $0.95 \pm 0.21$ & $0.94 \pm 0.23$ & $0.75 \pm 0.18^{*, * *, * * *}$ \\
\hline & Control & $0.93 \pm 0.33$ & $0.94 \pm 0.28$ & $0.93 \pm 0.32$ \\
\hline
\end{tabular}

Notes: Data are presented as mean \pm SD. Compared with control group, $* P<0.05$; compared with before treatment, $* * P<0.05$; compared with 4 weeks after treatment, $* * * P<0.05$.

Abbreviation: UK, urinary kallidinogenase.

automatic regulation of vasomotion, collectively aggravating ischemic cerebral damage. ${ }^{4}$ Meanwhile, vascular risk factors can predict the clinical development of cognitive dysfunction. Cerebral hypoperfusion can lead to major reductions in suboptimal delivery of high-energy nutrients to the brain, with lethal consequences to brain cells that participate in cognitive function. ${ }^{16-20}$ Thus, expanding the blood vessels, improving the cerebral blood supply, and restoring the neurological deficit are urgent for the patients with cerebral arterial stenosis.

UK is a positive regulatory substance in KKS consisting of kinins, kallikreins, and kininogens by producing kallidin and kinin, and has been identified to protect against ischemic brain injury through multiple signaling pathways, including anti-inflammation and antiapoptosis, and to promote angiogenesis and neurogenesis. ${ }^{21,22}$ UK could supply kallikrein to patients' salvages brain tissue by expanding blood vessels, recanalizing, and improving perfusion. The study on the efficacy of UK in stroke demonstrated that patients could benefit from it with $87 \%$ efficacy rate. ${ }^{2}$ In this study, we studied 45 patients with cerebral arterial stenosis that received UK could improve the cognitive status at 4 weeks after treatment than conventional treatment. Meanwhile, improved cognitive status in patients accompanied by $\mathrm{A} \beta 1-40$ decrease at 4 weeks after treatment and by $A \beta 1-42$ decrease at 8 weeks after treatment, possibly through decreased production or increased clearance of $A \beta$ fragments in cerebral tissues due to improved cerebral blood flow by UK. The findings in this study are consistent with most of the previous studies about UK for ischemic cerebral injury such as stroke. ${ }^{2}$ Furthermore, in this study, there is no side effect of hypotension, the most common adverse event of UK, on the patients using the new treatment method, which provides the evidence on the safety of UK in the application of patients with cerebral arterial stenosis.

Taken together, this study at first confirmed that UK could improve cognitive status and decrease $\mathrm{A} \beta \mathrm{s}$ to prevent the development and progression of $\mathrm{AD}$ and ischemic cerebral injury, which could represent an approach for patients with cerebral arterial stenosis. Due to the limitation of the short follow-up period, further investigation is needed in future.

\section{Conclusion}

In conclusion, UK successfully improved cognitive status in patients with cerebral arterial stenosis and decreased both $A \beta 1-40$ and A $\beta 1-42$ serum levels. Therapeutic strategy of the supplement of kallikreins to increase cerebral blood flow may prevent the development and progression of $\mathrm{AD}$ and ischemic cerebral injury.

\section{Acknowledgment}

We thank Xinyong Zhang, Zengjun Wang, Jingming Lu, and Guang Yang for assistance with patient recruitment.

\section{Disclosure}

The authors report no conflicts of interest in this work.

\section{References}

1. Wang YX, Chen Y, Zhang CH, et al. Study on the effect of urinary kallidinogenase after thrombolytic treatment for acute cerebral infarction. Eur Rev Med Pharmacol Sci. 2015;19(6):1009-1012.

2. Zhang C, Tao W, Liu M, Wang D. Efficacy and safety of human urinary kallidinogenase injection for acute ischemic stroke: a systematic review. J Evid Based Med. 2012;5(1):31-39.

3. Porcu P, Emanueli C, Kapatsoris M, Chao J, Chao L, Madeddu P. Reversal of angiogenic growth factor upregulation by revascularization of lower limb ischemia. Circulation. 2002;105(1):67-72.

4. Zhao L, Zhao Y, Zhang H. Effect of stent-assisted angioplasty on cognitive status and serum levels of amyloid beta in patients with intracranial and/or extracranial artery stenosis. Neuropsychiatr Dis Treat. 2015;11:471-475.

5. Wang PQ, Wang AP, Cao ZH, Wang P, Wang S, Zhang GB. The study of the distribution character of cerebral arterial stenosis in patients with ischemic cerebrovascular disease by means of 64 slices CT. Eur Rev Med Pharmacol Sci. 2015;19(12):2287-2292.

6. Chen CC, Chung CY, Lee TH, Chang WH, Tang SF, Pei YC. Increased risk of posterior circulation infarcts among ischemic stroke patients with cervical spondylosis. Neuropsychiatr Dis Treat. 2015;11:273-278.

7. Kolukisa M, Ozdemir Gültekin T, Eryigit Baran G, et al. One-year follow-up in patients with brainstem infarction due to large-artery atherothrombosis. Neuropsychiatr Dis Treat. 2015;11:379-384.

8. Roher AE, Esh C, Kokjohn TA, et al. Circle of willis atherosclerosis is a risk factor for sporadic Alzheimer's disease. Arterioscler Thromb Vasc Biol. 2003;23(11):2055-2062. 
9. Heinzel S, Liepelt-Scarfone I, Roeben B, et al. A neurodegenerative vascular burden index and the impact on cognition. Front Aging Neurosci. 2014;6:161-169.

10. Zhang X, Li L, Zhang X, et al. Prenatal hypoxia may aggravate the cognitive impairment and Alzheimer's disease neuropathology in APPSwe/PS1A246E transgenic mice. Neurobiol Aging. 2013;34(3): 663-678.

11. Al-Qazzaz NK, Ali SH, Ahmad SA, Islam S, Mohamad K. Cognitive impairment and memory dysfunction after a stroke diagnosis: a post-stroke memory assessment. Neuropsychiatr Dis Treat. 2014;10: 1677-1691.

12. Ghezzi L, Scarpini E, Galimberti D. Disease-modifying drugs in Alzheimer's disease. Drug Des Devel Ther. 2013;7:1471-1478.

13. Zhang LF, Zhou ZW, Wang ZH, et al. Coffee and caffeine potentiate the antiamyloidogenic activity of melatonin via inhibition of $\mathrm{A} \beta$ oligomerization and modulation of the Tau-mediated pathway in $\mathrm{N} 2 \mathrm{a}$ / APP cells. Drug Des Devel Ther. 2014;9:241-272.

14. Zhang F, Jiang L. Neuroinflammation in Alzheimer's disease. Neuropsychiatr Dis Treat. 2015;11:243-256.

15. Tuttolomondo A, Pecoraro R, Pinto A. Studies of selective TNF inhibitors in the treatment of brain injury from stroke and trauma: a review of the evidence to date. Drug Des Devel Ther. 2014;8:2221-2239.
16. de la Torre JC. Cerebral hemodynamics and vascular risk factors: setting the stage for Alzheimer's disease. J Alzheimers Dis. 2012;32: 553-567.

17. Silvestrini M, Viticchi G, Falsetti L, et al. The role of carotid atherosclerosis in Alzheimer's disease progression. J Alzheimers Dis. 2011;25:719-726.

18. Silvestrini M, Viticchi G, Altamura C, Luzzi S, Balucani C, Vernieri F. Cerebrovascular assessment for the risk prediction of Alzheimer's disease. J Alzheimers Dis. 2012;32:689-698.

19. Balestrini S, Perozzi C, Altamura C, et al. Severe carotid stenosis and impaired cerebral hemodynamics can influence cognitive deterioration. Neurology. 2013;80:2145-2150.

20. Gupta A, Iadecola C. Impaired A $\beta$ clearance: a potential link between atherosclerosis and Alzheimer's disease. Front Aging Neurosci. 2015;7: 115 .

21. Chen ZB, Huang DQ, Niu FN, Zhang X, Li EG, Xu Y. Human urinary kallidinogenase suppresses cerebral inflammation in experimental stroke and downregulates nuclear factor-kappaB. J Cereb Blood Flow Metab. 2010;30:1356-1365.

22. Albert-Weißenberger C, Sirén AL, Kleinschnitz C. Ischemic stroke and traumatic brain injury: the role of the kallikrein-kinin system. Prog Neurobiol. 2013;10(1-102):65-82.
Drug Design, Development and Therapy

\section{Publish your work in this journal}

Drug Design, Development and Therapy is an international, peerreviewed open-access journal that spans the spectrum of drug design and development through to clinical applications. Clinical outcomes, patient safety, and programs for the development and effective, safe, and sustained use of medicines are a feature of the journal, which

\section{Dovepress}

has also been accepted for indexing on PubMed Central. The manuscript management system is completely online and includes a very quick and fair peer-review system, which is all easy to use. Visit http://www.dovepress.com/testimonials.php to read real quotes from published authors.

Submit your manuscript here: http://www.dovepress.com/drug-design-development-and-therapy-journal 